\title{
Fibromuscular Dysplasia
}

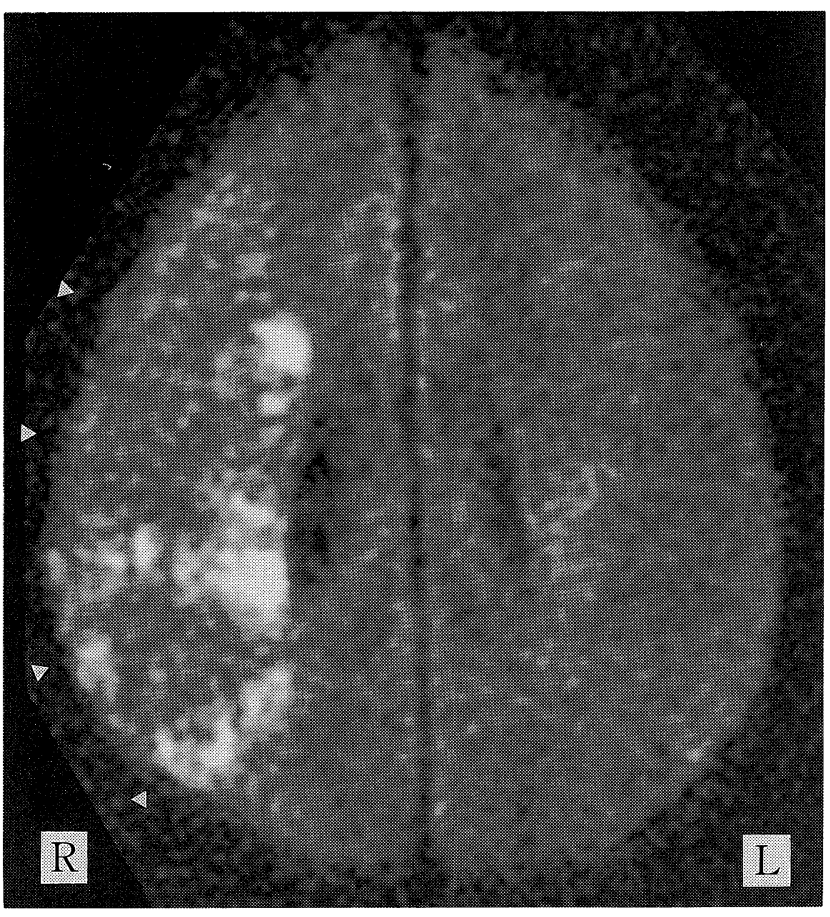

Figure 1. MR diffusion-weighted image demonstrates a highsignal intensity of mottled appearance occupying a large area of the right MCA territory (arrows).

Figure 2. A) A sclerotic arteriography of the right internal carotid artery discloses multisegmental narrowing of the extracranial portion. B) A similar multisegmental beeding pattern is apparent in the renal artery.

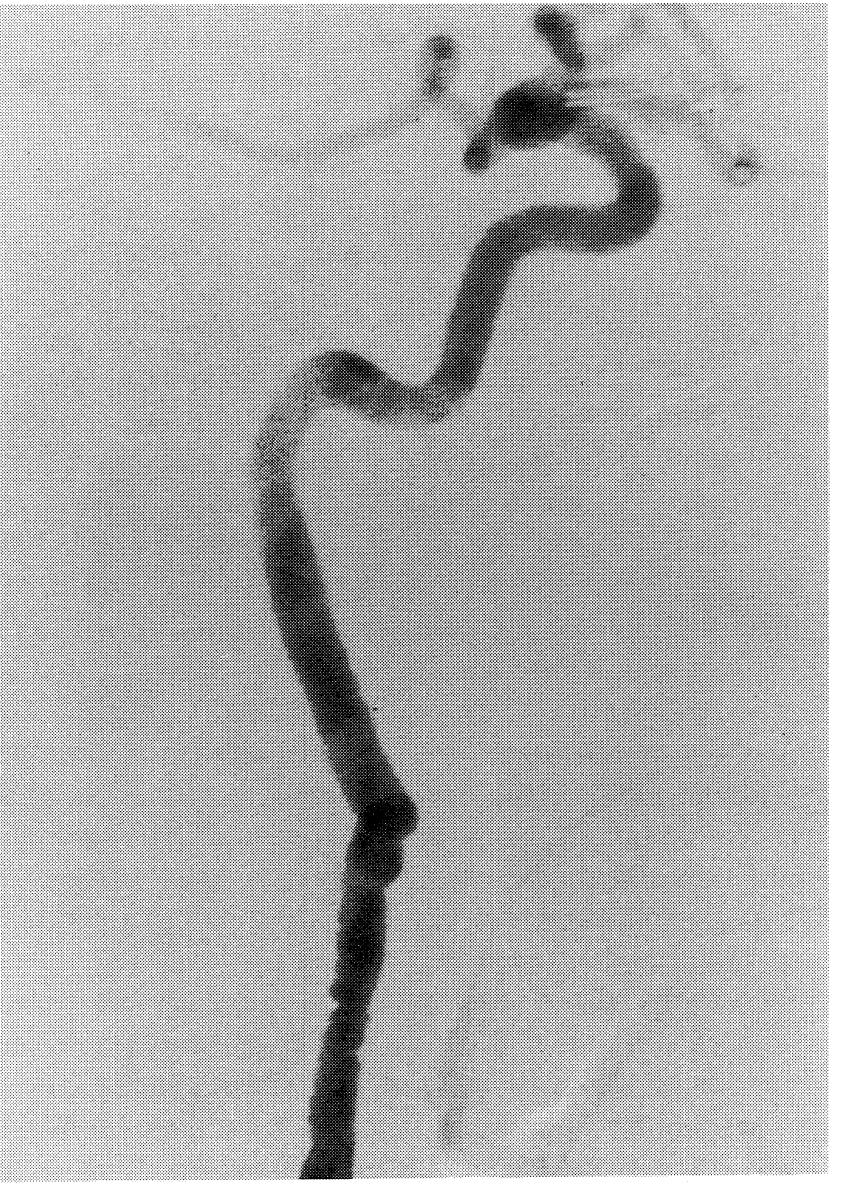

A

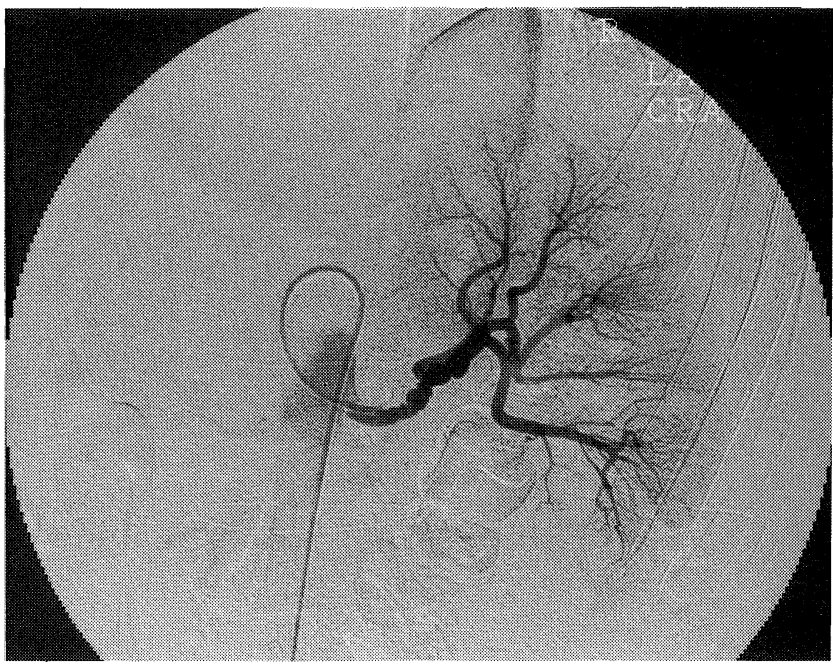

B 
Fibromuscular dysplasia (FMD) is a vasculopathy characterized by fibrous tissue proliferation, smooth muscle cell hyperplasia, and elastic fiber destruction, alternating with segments of mural thinning. Arterial involvements is segmental; and most commonly affected are the internal carotid arteries (IC) and the renal arteries. The cause of FMD is uncertain.

A 40-year-old man with a history of hypertension for the previous 20 years, and subarachnoid hemorrage due to ruptured aneurysm of right IC at age 25 developed transient left hemiparesis. General physical examination was unremarkable, but neurological examination showed minimal left hemiparesis with left-sided hyperreflexia and Babinski sign.

We treated him with antiplatelet drugs, and his neurological signs improved.

Diffusion-weighted MR images show a mottled high-signal intensity indicating ischemia in the territory of the right middle cereberal artery (Fig. 1). Angiography revealed a string-like appearance of both IC and renal arteries, being compatible with FMD (Fig. 2A, B).

Key words: diffusion-weighted MR images, angiography

Dai HATA

The Department of Neurosurgery, Hokkaido Kin-Ikyo Chuo Hospital Received for publication March 21, 2001; Accepted for publication May 17,2001

Reprint requests should be addressed to Dr. Dai Hata, the Department of Neurosurgery, Hokkaido Kin-Ikyo Chuo Hospital, 10-2-15-1 Fushiko, Higashi-ku, Sapporo, Hokkaido 007-0870 